\title{
Abordaje Terapéutico de Agrandamiento Gingival Influenciado por Ciclosporina y Nifedipino. Reporte de Caso.
}

\section{Therapeutic Approach of Gingival Enlargement Influenced by Cyclosporine and Nifedipine. Case Report.}

\author{
Ma. Angélica Michea ${ }^{1 *}$, Pablo Dittus ${ }^{2}$, Miguel Fernández ${ }^{1}$, Patricio Neira $^{1}$
}

1. Facultad de Odontología, Universidad de Chile. Santiago, Chile

2. Servicio de Salud Valdivia, Chile.

* Correspondencia Autor: María Angélica Michea. | Dirección: Sergio Livingstone 943 Independencia (8380492). Santiago de Chile. | Teléfono: +562 2978 1839. | E-mail: amichea@uchile.cl Trabajo recibido el 20/03/2020.

Aprobado para su publicación el 03/08/2020

\begin{abstract}
RESUMEN
Este reporte de caso muestra un paciente atendido en el Postítulo de Periodoncia de la Facultad de Odontología de la Universidad de Chile con diagnóstico de Agrandamiento Gingival influenciado por ciclosporina y nifedipino. El abordaje terapéutico consideró la fase sistémica, la fase higiénica con el tratamiento periodontal no quirúrgico para lograr la eliminación de la infección periodontal antes y después de la fase quirúrgica, y la fase de terapia de soporte periodontal. Se logró así la eliminación de los agrandamientos gingivales influenciados por ciclosporina y nifedipino.
\end{abstract}

\section{PALABRAS CLAVE:}

Agrandamiento gingival; Ciclosporina; Enfermedades periodontales; Transplante renal.

Int. J. Inter. Dent Vol. 13(3); 168-170, 2020.

ABSTRACT

This case report shows a patient attended in the Postgraduate Periodontics Program at the Faculty of Dentistry of the University of Chile with a diagnosis of Gingival Enlargement influenced by cyclosporine and nifedipine. The therapeutic approach considered the systemic phase, the hygienic phase with the non-surgical periodontal treatment to achieve the elimination of the periodontal infection before and after the surgical phase, and the phase of periodontal support therapy. Thus, the elimination of gingival enlargements influenced by cyclosporine and nifedipine was achieved.

KEY WORDS:

Gingival overgrowth; Cyclosporine; Periodontal diseases; Kidney transplantation.

Int. J. Inter. Dent Vol. 13(3); 168-170, 2020.

\section{INTRODUCCIÓN}

La indicación de terapia farmacológica para el tratamiento de una enfermedad puede tener efectos adversos en la cavidad oral. Así, algunos fármacos de la familia de los antiepilépticos (fenitoína), inmunosupresores (ciclosporina (Cs)), bloqueadores de canales de calcio (nifedipino, entre otros) y altas dosis de anticonceptivos orales se vinculan con la aparición de agrandamiento gingival $(A G)^{(1)}$. AG se define como el crecimiento exce-sivo de la encía debido a un incremento del tamaño de las células constituyentes o hipertrofia gingival, o por un incremento en el número de las células o hiperplasia gingival. La prevalencia reportada en pacientes consumidores de Cs y transplantados de riñón, varía entre un $25 \%$ y un $81 \%$ en función de la dosis, duración de la terapia o condición médica ${ }^{(2)}$. Según el esquema de Clasificación de las enfermedades y condiciones periodontales y peri-implantares de 2017, un escenario de AG derivado del consumo de un medicamento que se prescribió para tratar una patología, donde el efecto adverso es el AG, corresponde a un Agrandamiento gingival influenciado por medicamentos, que es parte de la Gingivitis inducida por biofilm dental ${ }^{(3)}$. La recopilación de la historia médica en conjunto con un acucioso examen clínico es suficiente para determinar la etiología, considerando que esté presente el medicamento que produce el AG como efecto adverso. La Cs es un inmunosupresor del sistema inmune, se indica para la prevención del rechazo de un órgano trasplantado o enfermedades autoinmunes y puede inhibir algu-nas de las funciones de los linfocitos T como el reconocimiento de antígenos, el procesamiento antigénico por macrógafos o la producción y liberación de interleuquina $1^{(4)}$. El nifedipino es un bloqueador de los canales de calcio indicado para la hipertensión arterial y la angina de pecho(5). Ambos medicamentos producen $A G$ como efecto adverso. Seymour identificó los factores predisponentes asociados con el desarrollo y la ex-presión de cambios gingivales influenciados por medicamentos ${ }^{(6)}$ : biofilm dental e inflamación gingival, edad, género, genética, tipo de medicamento, dosis, concentración (plasmática, tisular y salival) y duración de la terapia. Para dilucidar la etiopatogenia del AG, se ha indagado tanto en el incremento de la proliferación de fibroblastos gingivales con un aumento en la producción de colágeno ${ }^{(7)}$ como en la sobreexpresión de ciertas moléculas del tejido gingival, como el factor de crecimiento de tejido conectivo, el factor de crecimiento tisular beta uno, la interleuquina uno alfa, interleuquina seis $u$ ocho ${ }^{(8)}$. Dentro de las implicancias clínicas, el AG por sí mismo genera una mayor dificultad para poder llevar a cabo una efectiva higiene oral. Los AG provocan la acumulación adicional de biofilm dental supra y subgingival, mayor inflamación y sangrado gingival, por lo tanto, el potencial para que se desarrolle una enfermedad periodontal(3). El crecimiento de la encía puede provocar migración dentaria patológica y problemas estéticos para el paciente, existiendo variaciones en el patrón clínico intra e interpacientes. En relación a la prevención y el tratamiento, el manejo del AG influenciado por medicamentos puede abordarse considerando la prevención de la inflamación gingival inducida por biofilm dental, eliminación de la infección periodontal cuando ya está establecida en el paciente, terapia quirúrgica para la remoción de los AG y terapia de soporte periodontal ${ }^{(9)}$. El objetivo de este reporte de caso es dar a conocer el caso clínico de un paciente con diagnóstico de AG influenciado por ciclosporina y nifedipino y cómo este enfoque de abordaje terapéutico es relevante ya que permitió eliminar los 
AG y mantener una estabilidad periodontal durante la terapia de soporte periodontal.

\section{CASO CLÍNICO}

Paciente género masculino, 27 años, acude al Postítulo de Periodoncia de la Facultad de Odontología de la Universidad de Chile. Antecedentes mórbidos: monorreno congénito, anomalía en la cual el paciente solo tiene un riñón desde el nacimiento. No existían antecedentes familiares pre existentes de esta condición. No conservó una buena función renal y evolucionó a una insuficiencia renal crónica, con diálisis por 4 años (2006 - 2010), siendo sometido a trasplante renal en 2010 , quedando con terapia inmunosupresora de Cs $25 \mathrm{mg}$. cada 12 horas (consumo por al menos 6 años). Hipertensión arterial controlada con nifedipino $20 \mathrm{mg}$. cada 12 horas y diltiazem $60 \mathrm{mg}$. 1 vez al día. Desarrolló diabetes mellitus post trasplante renal (2014), controlada con metformina $850 \mathrm{mg}$. cada 8 horas. Hemoglobina glicosilada de $6.5 \%$, valor entregado durante el periodo de anamnesis, cuando se le solicitó traer sus exámenes complementarios. Paciente en controles médicos regulares con nefrólogo, quien solicitaba periódicamente creatininemia, nitrógeno ureico y orina completa, entre otros exámenes, para controlar función renal y control metabólico. Sin tratamiento periodontal previo. Al examen clínico intraoral (Figura 1) se observa AG de 6 años de evolución, maxilar y mandibular generalizado, indoloro, fibrótico en las zonas posteriores y palatinas y friable en el sextante 2 y 5 , en donde el AG cubre más del $75 \%$ de la corona clínica. Presenta migración dentaria patológica del diente 1.1 hacia vestibular, cálculo dental generalizado supragingival y subgingival, sangrado al sondaje $>90 \%$ y pseudosacos periodontales. Los AG tienen base pedunculada en sextante 2 y 5 y a la presión digital presentaban supuración. Se solicitó radiografía panorámica. Índice de Placa de $100 \%$. El paciente fue informado del tratamiento al que sería sometido y firmó el consentimiento informado relacionado con el tratamiento y con el reporte de su caso. El plan de tratamiento se detalla a continuación:

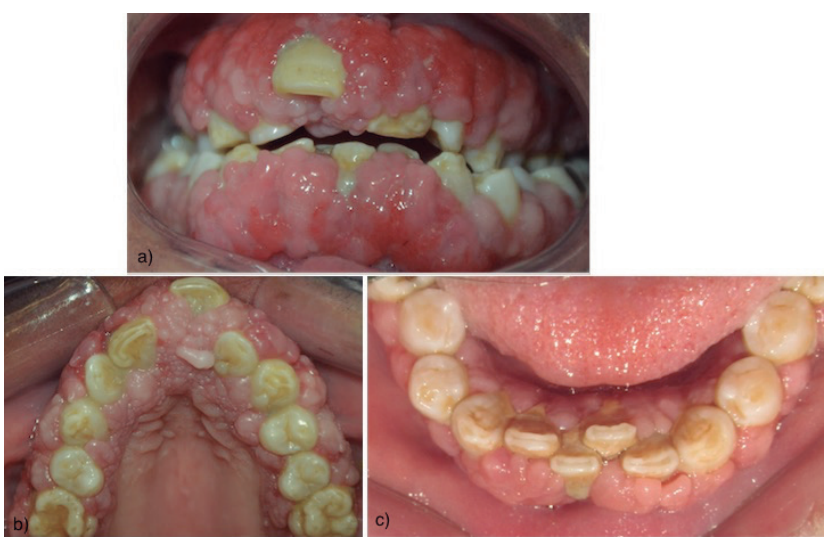

Figura 1. Fotografía intraoral a) Frontal. b) Oclusal superior. c) Oclusal inferior

Fase sistémica: Recopilación de patologías concomitantes y sus medicamentos prescritos. Se realizó interconsulta a nefrólogo, solicitando indicaciones respecto de suspensión o indicación de medicamentos y los resguardos necesarios ante la condición médica del paciente. Obtención de pase médico, se consideró con control metabólico aceptable, con la indicación de profilaxis antibiótica de amoxicilina 2 gramos 1 hora previo a todo tratamiento periodontal no quirúrgico y quirúrgico. No se realizó cambio por otro antibiótico, ya que éste fue el prescrito por el nefrólogo.

Fase higiénica: Esta fase se abordó en dos etapas, previo a la fase quirúrgica (etapa 1) y posterior a la fase quirúrgica (etapa 2). Fase higiénica etapa 1: Instrucción de técnica de cepillado de barrido con cepillo dental de filamentos suaves y redondeados (Curaprox CS $5460^{\circledR}$ ), frecuencia de cepillado de 3 veces al día, después de las comidas, hilo dental (Oral B Satin Tape ${ }^{\circledR}$ ), cepillos interdentales $0,6 \mathrm{~mm}$. (Dentaid Interprox Plus ${ }^{\circledR}$ ) después de las comidas, pasta dental con triclosán (Colgate total $12^{\circledR}$ ) y refuerzo de cepillado en cada sesión. Destartraje supra y subgingival en dos sesiones, colutorio de clorhexidina $0,12 \%$ por dos semanas. No se realiza periodontograma.

Fase quirúrgica: Esta fase también se abordó en dos etapas. Fase quirúrgica etapa 1: Dos semanas posteriores al destartraje realizado en la fase higiénica etapa 1, se realizó gingivectomía maxilar y mandibular (Figura 2). Técnica anestésica infiltrativa al $2 \%$, se midió con sonda periodontal Carolina del Norte los pseudosacos periodontales y se hizo el marcaje externo. Se realizó incisión discontinua en dirección coronal en un ángulo de $45^{\circ}$ con bisturí \#15c, bisel interno, y se creó un contorno marginal festoneado. Se retiró los excesos de tejido gingival vestibular e interproximal con tijera quirúrgica, pinzas y curetas Gracey. Finalmente se colocó una gasa con suero fisiológico en los tejidos para realizar hemostasia, con presión por 5 minutos. Se indicó clorhexidina $0,12 \%$ por dos semanas y paracetamol 1 gramo cada 8 horas (no nefrotóxicos) en caso de malestar postoperatorio. Se controló la presión arterial y saturación de oxígeno por parte de un técnico en enfermería durante el procedimiento quirúrgico. Se realizó un control post quirúrgico a los dos días, observándose un cambio de la arquitectura y disminución de la inflamación gingival (Figura 3).

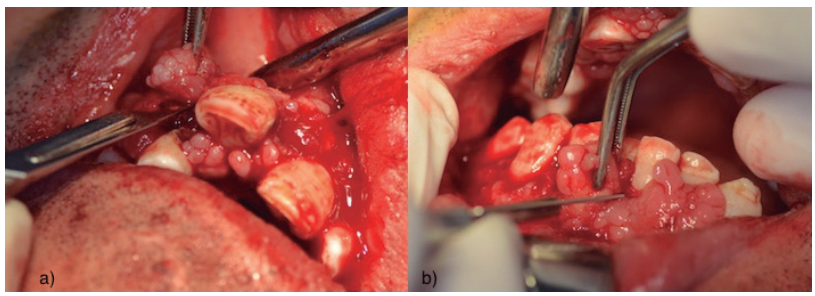

Figura 2. Gingivectomía encía a) Maxilar. Nótese vestibularización diente 1.1. b) Mandibular

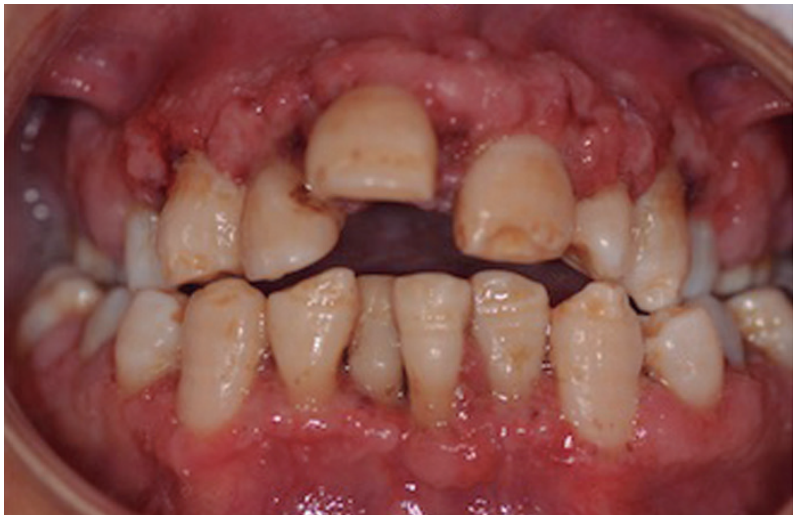

Figura 3. Control 2 días post quirúrgico

Fase higiénica etapa 2: Se continuó con el control de biofilm dental en forma periódica por un mes. Al segundo mes post cirugía, cuando ya no estaban presentes los AG ni los pseudosacos, se realizó el periodontograma, con el cual el paciente tuvo un diagnóstico de periodontitis etapa II generalizada grado B. El paciente presentó p`érdida de inserción clínica en molares superiores e inferiores y en sextante 2 de hasta $4 \mathrm{~mm}$. Se mantuvo la radiografía panorámica. Se realizó pulido y alisado radicular por cuadrantes.

Fase quirúrgica etapa 2: Cuatro semanas posterior al último pulido radicular, se realizó gingivoplastía bajo anestesia infiltrativa al $2 \%$ con fresas diamantadas de alta velocidad. Se observó una recidiva del AG en la papila dental entre diente 1.3 y 1.2 (Figura $4 a$ ). Se repite el tratamiento con pulido y alisado radicular.

Fase de Terapia de Soporte Periodontal: Luego de la eliminación de la infección periodontal inicial del paciente mediante la fase higiénica y quirúrgica en las cuales se logró un aceptable control de biofilm dental (Índice de Placa $<30 \%$ ) y la eliminación de los AG, se consideró mantener al paciente en Terapia de Soporte Periodontal, con controles periódicos cada 3 meses para mantener el estado de salud gingival logrado hasta el momento (sangrado al sondaje $<15 \%$ ). En esta etapa se observó una reducción de la vestibularización del diente 1.1 (Figura 4b). El paciente no tuvo sensibilidad dental posterior a todo el tratamiento realizado.

\section{DISCUSIÓN}

Los reportes de caso existentes en relación al AG influenciado por Cs enfatizan tanto el tratamiento periodontal no quirúrgico como el tratamiento periodontal quirúrgico, recomendando una adecuada y oportuna instrucción de higiene oral para el autocontrol de biofilm dental y una terapia de soporte periodontal para evitar la recidiva. El presente reporte de caso no dista de lo que ya se ha presentado en otros reportes de $\operatorname{casos}^{(10,11,13,15)}$, el manejo clínico puede ser similar a lo reportado, no obstante se desea mostrar paso a paso el plan de tratamiento escogido, el cual obedece al siguiente análisis: Se inicia con la fase sistémica, con recopilación de la información médica, los medicamentos prescritos para las patologías asociadas y la identificación 


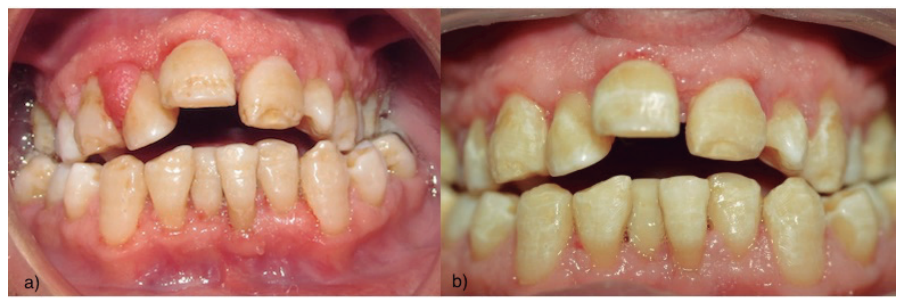

Figura 4. a) 3 meses post cirugía. Nótese recidiva de $A G$ en papila diente 1.3 - 1.2. b) Terapia de Soporte Periodontal (6 meses). Eliminación de Agrandamiento Gingival, palatinización diente 1.1

de las cirugías a las que fue sometido el paciente, quien presenta una condición médica de cuidado: monorreno congénito, insuficiencia renal crónica, transplantado renal, diabetes mellitus secundaria a transplante e hipertensión arterial. Es necesario que el médico tratante esté informado mediante una interconsulta del plan de tratamiento dental que se planificó para el paciente, para que así informe de los cuidados pre y post tratamiento periodontal necesarios. Es ya conocida la relación entre el estado periodontal y la condición sistémica de los pacientes ${ }^{(12)}$. Tanto en los casos de AG leves como en los AG moderados y severos se propone continuar con la fase higiénica etapa 1, en la cual se realiza instrucción de higiene oral para lograr un adecuado control de biofilm. El objetivo fue que el paciente redujera los índices de placa bacteriana mediante el control mecánico, con la ayuda tanto de la clorhexidina al $0,12 \%$ por dos semanas, como del dentífrico con triclosán para reducir la carga bacteriana. Se justificó el uso de clorhexidina al $0,12 \%$ al inicio del tratamiento debido a sus AG de larga data, generalizados, donde el tratamiento mecánico por sí solo no iba a controlar la infección de modo efectivo, específicamente en los pseudosacos, que eran la mayoría. El índice de placa disminuyó de un $100 \%$ a un valor $<30 \%$ durante la terapia de soporte periodontal. Cuando no se logre un óptimo control manual de biofilm dental o haya una alteración de la motricidad del paciente, se puede optar por la alternativa de cepillo eléctrico y uso de colutorios en base a clorhexidina $0,12 \%$ o cloruro de cetilpiridino $0,05 \%$ para obtener beneficios adicionales. La higiene interdental debe llevarse a cabo con cepillos interdentales de preferencia. En este caso se utilizaron en conjunto con la seda dental. Luego se procedió con el destartraje supra y subgingival con instrumentos ultrasónicos. El objetivo de esta etapa fue llevar a cabo acciones clínicas efectivas para la eliminación del biofilm dental supra y subgingival, reduciendo así la inflamación gingival, favoreciendo así la reducción del AG asociado a biofilm dental. Se consideró todos los factores locales que predisponían a la acumulación de biofilm dental, por ejemplo, pulido y ajuste de restauraciones mal contorneadas.

Luego de cumplida la fase higiénica etapa 1 , se llevó a cabo la fase quirúrgica etapa 1 , con la técnica de gingivectomía ${ }^{(13)}$. Las indicaciones de gingivectomía para los casos de $A G$ son ${ }^{(14)}$ : Cuando el tratamiento periodontal no quirúrgico no resuelve los $A G$ o en $A G$ que interfieren con la masticación, habla o estética. El objetivo de esta primera intervención quirúrgica fue la escisión de los AG para favorecer una mejor higiene oral por el paciente, mejor función masticatoria y del habla, además de mayor visibilidad de diente y de los tejidos periodontales para el especialista. Posteriormente, se realizó la fase higiénica etapa 2, donde se realizó un tratamiento periodontal no quirúrgico por cuadran-tes orientado a tratar la periodontitis etapa II generalizada grado $B$, con pérdida de inserción que los $A G$ enmascaraban, realizando el pulido y alisado radicular por cuadrantes. De ser necesario, una fase quirúrgica etapa 2 con gingivoplastía, puede ser realizada para restaurar el contorno gingival. El tratamiento periodontal quirúrgico puede ser realizado con bisturí, electrobisturí, láser o tijeras quirúrgicas en la gingivectomía y con láser y fresas diamantadas de alta velocidad para la gingivoplastía ${ }^{(15)}$. Una terapia de soporte periodontal permite la mantención de la salud periodontal lograda en las fases anteriores, el refuerzo del control de biofilm dental supragingival por parte del paciente y así reducir el riesgo de inflamación gingival y AG. La recurrencia de los AG puede darse debido no solo a la variable del medicamento que induce el $A G$ sino que también por un tratamiento periodontal no exitoso cuando hay un mal control de biofilm dental( ${ }^{(6)}$. En este caso se reforzó el control de biofilm frecuentemente durante el tratamiento periodontal no quirúrgico. Se destaca que hubo una palatinización del diente 1.1 luego de la fase quirúrgica etapa 1, el cual tenía migración dentaria patológica. Este cambio en la posición inicial del diente 1.1 producto de la disrupción de las fuerzas que mantenían el diente en posición, cuyo origen fue la presión de los tejidos blandos del AG circundante y la pérdida de inserción clínica, provocó su vestibularización. Una adecuada terapia de soporte periodontal es fundamental para evitar la recidiva de la enfermedad periodontal, idealmente cada 3 meses para reforzar la higiene oral, controlando los factores etiológicos locales, reforzando la adherencia del paciente al tratamiento para mantener la estabilidad periodontal lograda con el tratamiento periodontal no quirúrgico y quirúrgico. Sin lugar a dudas la calidad de vida del paciente también se ve mejorada. Este enfoque de abordaje terapéutico puede ser aplicado en otros pacientes con similitudes clínicas.

\section{CONCLUSIONES}

El Agrandamiento gingival influenciado por medicamentos, que es parte de la Gingivitis inducida por biofilm dental es una condición clínica cuya prevención y estrategias de tratamiento están basadas en la evidencia disponible y se aplican con el objetivo de mejorar la condición periodontal del paciente en el tiempo. La fortaleza de este reporte de caso es que se realiza un plan de tratamiento ordenado, paso a paso, fundamentando cada etapa. Una propuesta adecuada y ordenada de tratamiento periodontal no quirúrgico y quirúrgico en pacientes trasplantados que consumen ciclosporina y nifedipino puede reducir los agrandamientos gingivales y la inflamación gingival, mejorando además la calidad de vida del paciente y pudiendo lograr una estabilidad periodontal durante la terapia de soporte periodontal.

\section{RELEVANCIA CLÍNICA}

El abordaje terapéutico del Agrandamiento gingival influenciado por medicamentos implica conocer tanto las técnicas quirúrgicas para la eliminación del tejido gingival que ha crecido en exceso como consecuencia del medicamento, como que el biofilm dental tiene un rol en el inicio y mantención de la inflamación gingival que puede provocar una recidiva. El control del biofilm dental, tratamiento periodontal no quirúrgico, quirúrgico y la terapia de soporte periodontal son las alternativas terapéuticas.

Se justifica este caso para dar a conocer el enfoque del abordaje terapéutico realizado a este paciente.

La consecuencia práctica es que puede ser un modelo terapéutico para su aplicación en otros pacientes con similitudes clínicas.

\section{CONFLICTO DE INTERESES}

Los autores declaran no tener algún conflicto de interés.

\section{Bibliografía}

1. Brown R, Arany P. Mechanism of drug-induced gingival overgrowth revisited: a unifying hypothesis. Oral Dis. 2015;21:e51-61.

2. Seymour RA, Jacobs DJ: Cyclosporin and the gingival tissues. J Clin Periodontol. 1992;19: 1-11

3. Murakami S, Mealey BL, Mariotti A, Chapple ILC. Dental plaque-induced gingival conditions. J Periodontol. 2018;89(Suppl 1):S17-S27.

4. Ponnaiyan D, Jegadeesan V. Cyclosporine A: Novel concepts in its role in druginduced gingival overgrowth. Dent Res J. 2015;12(6):499-506.

5. Vidal F, de Souza RC, Ferreira DC, Fischer RG, Gonçalves LS. Influence of 3 calcium channel blockers on gingival overgrowth in a population of severe refractory hypertensive patients. J Periodontal Res. 2018;53(5):721-26.

6. Seymour RA, Smith DG. The effect of a plaque control programme on the incidence and severity of cyclosporine- induced gingival changes. J Clin Periodontol. 1991;18(2):107-10

7. Schincaglia GP, Forniti F, Cavallini R, Piva R, Calura G, del Senno L. Cyclosporin-A increases type I procollagen production and mRNA level in human gingival fibroblasts in vitro. J Oral Pathol Med. 1992;21(4):181-5.

8. Trackman PC, Kantarci A. Molecular and clinical aspects of drug-induced gingival overgrowth. J Dent Res. 2015;94(4):540-6.
9. Beaumont J, Chesterman J, Kellett M, Durey K. Gingival overgrowth: Part 1: aetiology and clinical diagnosis. Br Dent J. 2017;222(2):85-91.

10. Campolo A, Nuñez L, Romero $P$, Rodriguez A, Fernández M, Donoso $F$. Agrandamiento gingival por ciclosporina: reporte de un caso. Rev Clin Periodoncia Implantol Rehabil Oral. 2016;9(3):226-30.

11. Paixão CG, Sekiguchi RT, Saraiva L, Pannuti CM, Silva HT, Medina-Pestana $J$, et al. Gingival overgrowth among patients medicated with cyclosporin A and tacrolimus undergoing renal transplantation: a prospective study. J Periodontol. 2011;82(2):251-8.

12. Chan S, Pasternak GM, West MJ. The place of periodontal examination and referral in general medicine. Periodontol 2000. 2017;74(1):194-9.

13. Chesterman J, Beaumont J, Kellett M, Durey K. Gingival overgrowth: Part 2: management strategies. Br Dent J. 2017;222(3):159-65.

14. Claffey N, Polyzois I, Ziaka P. An overview of nonsurgical and surgical therapy. Periodontol 2000. 2004;36:35-44.

15. Mavrogiannis M, Ellis JS, Thomason JM, Seymour RA. The management of drug- induced gingival overgrowth. J Clin Periodontol. 2006;33:434-9. 